\title{
Complete Valorization of Olive Mill Wastewater through an Integrated Process for Poly-3-hydroxybutyrate Production
}

\author{
Vito Emanuele Carofiglio ${ }^{1}$, Roberta Romano ${ }^{2}$, Maurizio Servili ${ }^{3}$, Antonio Goffredo ${ }^{1}$, Pietro Alifano ${ }^{2}$, Gianluca \\ Veneziani $^{3}$, Christian Demitri ${ }^{4}$, Domenico Centrone ${ }^{1}$ and Paolo Stufano ${ }^{1}$ \\ 1. EggPlant S.r.l., via Don Minzoni 27, Polignano a Mare (Bari) 70044, Italy \\ 2. Department of Biological and Environmental Sciences and Technologies (DiSTeBA), University of Salento, via Monteroni, \\ Campus Ecotekne, Lecce 73100, Italy \\ 3. Department of Agricultural, Food and Environmental Sciences, University of Perugia, via S. Costanzo, Perugia 06126, Italy \\ 4. Department of Engineering for Innovation, University of Salento, via Monteroni, Campus Ecotekne, Lecce 73100, Italy
}

\begin{abstract}
An economical and environmental sustainability of bioplastic production is dependent on the use of low cost and waste C-sources as raw materials. OMW (Olive Mill Wastewater) with its high organic load represents a dangerous polluting waste. Herein the authors present an integrated process for the simultaneous recovery of polyphenols, high value natural antioxidants, production of PHAs (polyhydroxyalkanotes), thermoplastic bio-polymers, in particular of PHB (poly-3-hydroxybutyrate) starting from OMW. The combination of membrane filtration and bacterial digestion of OMW resulted in very high yields of polyphenols $(32.5 \mathrm{~g} / \mathrm{L})$ and PHB $(31.4 \mathrm{mg} / \mathrm{L} \cdot \mathrm{h})$ if compared with the state of the art. These results make the technical approach described here effective for reducing the polluting effect of OMW and maximizing the valuable product yield. Moreover the process is readily suitable for an industrial scale PHB production from OMW.
\end{abstract}

Key words: Poly-3-hydroxybutyrate, olive mill wastewater, polyphenol, Azotobacter vinelandii UWD, membrane filtration.

\section{Introduction}

Global dependence on petroleum-derived plastics has increased considerably over the years.

However, the rapid depletion of crude oil and the mounting apprehension about the environmental effects of synthetically produced materials has prompted much interest in biologically derived polymers, particularly of the biodegradable class. Modern biotechnology has made it possible to manipulate these biopolymers to suit human needs. Among the various biodegradable polymers, a class that is drawing considerable attention is the PHAs (polyhydroxyalkanoates).

PHAs are bacterial polymers that are formed as

Corresponding author: Vito Emanuele Carofiglio, Ph.D., Dr., research fields: waste valorization, microbial fermentation, biopolymer production. E-mail: vitoemanuele@eggplant.it. naturally occurring storage polyesters by a wide range of microorganisms usually under unbalanced growth conditions. They offer similar characteristics to conventional plastics and they can be degraded at high rates to carbon dioxide and water by numerous bacterial strains, making PHAs attractive substitutes to petrochemical thermoplastics [1]. The most common representative of PHAs is the homopolymer PHB (poly-3-hydroxybutyrate).

There are a number of literature reviews on the selection of suitable carbon sources for efficient PHA production, for which the total cost of bioprocessing must meet economic requirements. The most frequently reported factor that influences the price of PHA is the cost of the carbon source [2]. Given the limits on agricultural capacity and the increasing demand for crops as bioenergy resources, researchers need to seek 
ways of using non-food crops for biodegradable production. Recent research focus therefore has been concentrated on the utilization of inexpensive renewable raw material for PHA production such as waste fats and oils, molasses, cheese whey, urban, farm and industrial wastewater [3-5].

In recent years, the use of organic waste has gained considerable attention for alternative substrate for PHA production. The liquid effluent of olive oil process named OMW has been considered as a promising no-cost alternative substrate for PHA production [6]. OMWs are characterized by undesirable color and odor, acidic $\mathrm{pH}$, high salt concentration and polyphenolic content. Generally, OMW pH ranges from 4.5 to 6 and includes $3-16 \%$ organic compounds, of which $1-8 \%$ are sugars, $1.2-2.4 \%$ are nitrogen-containing compounds [7]. Consequently the environmental impact of OMW is dramatic, as its COD (chemical oxygen demand) value generally ranges between 40 and $195 \mathrm{~g} / \mathrm{L}$ [8], that is $100-200$ times higher than urban wastewater. This high COD value represents a hidden source of value, as all the chemicals can be separated from the waste stream and residual organic content used as raw-material for bio-polymers production. Olive oil is mainly produced in the Mediterranean countries, although other producers, such as Argentina, Australia and Chile, are facing the toxic effects of OMW [9]. The major olive oil producing countries are Spain, Italy, and Greece, with a production of $1,150,560$, and 370 thousand tons annually, respectively, followed by Tunisia and Turkey, with an annual production of 160 thousand tons each [10]. The three-phase centrifugation system, largely used in Italy, provides a dilution of the malaxed pastes producing $50-90 \mathrm{~L}$ of OMWs $/ 100 \mathrm{~kg}$ of olive pastes [7].

Thus, since OMWs are generated in huge quantities in a short period of time, their disposal constitutes a serious environmental problem for olive-oil producing countries. OMWs are significant sources of environmental pollution because they inhibit seed germination and early plant growth, alter soil characteristics, and create reducing conditions, affecting microbial diversity in soil [11]. This is mainly due to the presence of phenolic compounds, which make OMW resistant to biological degradation and capable of inhibiting microbial growth [12]. Several approaches have been developed to achieve the reduction of OMW pollutant load including membrane separation techniques, which allow the recovery of antioxidant compounds [13], ultra-pure water [14] and anaerobic digestion [15], that when coupled with an aerobic fermentation can afford PHA production [16, 17].

The membrane separation approach is based on size-exclusion membrane filtration techniques that allow the recovery of valuable chemicals from the waste streams. Different studies have been focused on treating and purifying high COD wastewater streams, usually based on membrane filtration techniques with a final RO (reverse osmosis) step to generate ultra-pure water, which can be re-used in the aerobic fermentation or collected for other applications. The extracted organic matter may be used to make compostable materials or for bio-gas production by microbial digestion. Alternatively the organic content of OMW can be used as primary carbon source for PHA production in a two stage (anaerobic-aerobic) bacterial fermentation by mixed or single strain $[16,18,19]$. The family Azotobacteriace, aerobic nitrogen-fixing bacteria, is characteristic of PHA accumulation. Nutrient-regulated hyperaccumulation of PHB has been reported in Azotobactervinelandii UWD (A. vinelandii UWD), which produced PHAs during growth on a variety agricultural and industrial wastewater [20].

Herein the authors report a novel combined approach for the complete valorization of OMW by integrating in a unique process a membrane treatment of the waste stream and the production of PHA by microbial digestion. Coupling the membrane filtration system with the bacterial PHA fermentation is highly advantageous since the DROC (dephenolized reverse 
osmosis concentrate) obtained by subsequent membrane treatments represents a controlled carbon-source for the bacterial PHA production. DROC has indeed been used as sole substrate for anaerobic fermentation with a mixed mesophilic culture and then subjected by a single bacterial strain, A. vinelandii UWD, with high PHB accumulation potential.

The work done has been aimed to find the optimal conditions and process design for PHB production from OMW, mainly in terms of reducing the volumes used for PHB bacterial production and maximizing the bio-polymer yield. At the same time polyphenols and ultrapure water are recovered by the membrane treatments, for a complete valorization of the starting OMW.

\section{Materials and Methods}

\subsection{Olive Mill Wastewater}

OMW was obtained from Moraiolo Cv. olives. The drupes were processed by the extraction system of Az. Agricola Petesse (Foligno, Perugia, Italy) equipped with a X4 three-phase decanter centrifuge (Alfa Laval, Tavarnelle V.P., Florence, Italy) based on horizontal separation technology with a working capacity of 2 ton $/ \mathrm{h}$. The analysis of fresh OMW showed a COD of $81.6 \mathrm{~g} / \mathrm{L}$, total $\mathrm{N}$ of $0.93 \mathrm{~g} / \mathrm{L}$, a total reducing sugars content of $0.74 \%$ with a $\mathrm{pH}$ value of 5.00 . The OMW samples were maintained under refrigeration at $4{ }^{\circ} \mathrm{C}$.

\subsection{Membrane System and DROC Production}

A ROC (reverse osmosis concentrate) was obtained by membrane treatments of OMWs within $24 \mathrm{~h}$ of their extraction. After their collection OMWs were enzymatically treated, by depolymerizing pectinase from Aspergillus niger (BIODEP, Biotec S.r.1.). In particular this enzymatic preparation was added at 500 grams per ton of fresh OMW and made to act at room temperature $\left(20{ }^{\circ} \mathrm{C}\right)$ for $12 \mathrm{~h}$. The following membrane treatments were microfiltration, ultrafiltration and reverse osmosis. The first step afforded a concentrate and microfiltration permeate (MFP), using tubular membranes made of organic polymers covered with polypropylene, which was further subjected to the following filtration steps as described by Servili et al. [7]. The industrial plant and all the membrane were purchased from Permeate S.r.1..

The dephenolization process was performed, using activated carbon (Filtercarb SA 1000, CarboitaliaS.r.1.), as follow: $3 \%$ and $10 \%$ of activated carbon was added respectively to MFP and ROC into a glass vessel and mixed with a Lab-Shaker (Adolf Kühner AG Birsfelden Basel) at $170 \mathrm{rpm}$ for $30 \mathrm{~min}$ to allow the total adsorption of phenols, then the activated carbon was repeatedly filtered through a filter paper using büchner funnel until its complete recovery.

\subsection{Streams Characterization}

\subsubsection{Chemical Oxygen Demand}

COD values $(\mathrm{mg} / \mathrm{L})$ before and after the membrane treatments were determined according to the official standard methods [21].

\subsubsection{Total Reducing Sugars}

Fehling's test was used to detect total reducing sugars content in OMW, MFP, ROC [22].

\subsubsection{Polyphenols}

Phenolic compounds were evaluated by SPE (solid phase extraction) according to Servili et al. [23]. The SPE was performed as follows: $1 \mathrm{~mL}$ of the sample (OMW, MFP, ROC and DROC) was loaded onto a high-load C18 cartridge (Bond Elut Jr-C18, 1,000 mg, Agilent Technologies), and the phenolic compounds were eluted with methanol $(50 \mathrm{~mL})$. After solvent removal under vacuum at $30{ }^{\circ} \mathrm{C}$, the residue was redissolved in $5 \mathrm{~mL}$ of methanol and then evaporated to dryness under a nitrogen flow.

The phenolic extract was recovered and then dissolved in methanol $(1 \mathrm{~mL})$ for HPLC (high performance liquid chromatography) analysis that was performed using an Agilent Technologies system model 1,100 consisting of a vacuum degasser, a quaternary pump, an autosampler, a thermostatted 
column compartment, a DAD (diode array detector), and a FLD (fluorescence detector), controlled by ChemStation (Agilent Technologies) and used for the elaboration of chromatographic data. A Spherisorb ODS-1 column was used to evaluate the phenolic compounds, the mobile phase consisted of $0.2 \%$ acetic acid ( $\mathrm{pH}$ 3.1) in water (solvent A)/methanol (solvent B) at a flow rate of $1 \mathrm{~mL} / \mathrm{min}$, and the gradient changed as follows: $95 \%$ A for $2 \mathrm{~min}, 75 \% \mathrm{~A}$ in $8 \mathrm{~min}$, $60 \% \mathrm{~A}$ in $10 \mathrm{~min}, 50 \% \mathrm{~A}$ in $16 \mathrm{~min}$, and $0 \% \mathrm{~A}$ in 14 $\min$ and maintained for $10 \mathrm{~min}$. Following the re-equilibration of the initial condition, equilibration was reached in $13 \mathrm{~min}$; the total running time was 73 min. All phenolic compounds, except lignans, which were detected by FLD, operated at an excitation wavelength of $280 \mathrm{~nm}$ and emission at $339 \mathrm{~nm}$, and DAD at $278 \mathrm{~nm}$.

\subsection{Microorganism}

Azotobactervinelandii UWD (strain ATCC 12837) was purchased from American Type Culture Collection. This strain is a mutant derivative of strain UW, and is defective in the respiratory oxidation of NADH. The mutation caused an overproduction of PHA. The microorganism was maintained in Burk's $\mathrm{N}$-free medium slant containing (g/L): $\mathrm{K}_{2} \mathrm{HPO}_{4}, 6.4 \mathrm{~g}$; $\mathrm{KH}_{2} \mathrm{PO}_{4}, 1.6 \mathrm{~g} ; \mathrm{NaCl}, 2.0 \mathrm{~g} ; \mathrm{MgSO}_{4} \cdot 7 \mathrm{H}_{2} \mathrm{O}, 2.0 \mathrm{~g}$; $\mathrm{CaSO}_{4} \cdot 2 \mathrm{H}_{2} \mathrm{O}, 0.5 \mathrm{~g} ; \mathrm{NaMoO}_{4} \cdot 2 \mathrm{H}_{2} \mathrm{O}, 0.01 \mathrm{~g}$; ferric citrate, $0.02 \mathrm{~g}$; glucose, $10.0 \mathrm{~g}$; distilled water, $1 \mathrm{~L}$. The $\mathrm{pH}$ was adjusted to 7.2 using $0.1 \mathrm{~N} \mathrm{NaOH}$. Burk's solid medium was formulated by adding $16.0 \mathrm{~g} / \mathrm{L}$ of agar-agar. All the products were purchased by Sigma Aldrich and J.T. Backer.

\subsection{Anaerobic and Aerobic Fermentation}

DROC and MFP were digested under anaerobic condition. MFP was previously diluted with sterile distilled water at a concentration of $60 \% \mathrm{v} / \mathrm{v}$, recovered by membrane filtration. The anaerobic digestion was carried out by laboratory-scale glass bioreactor with $500 \mathrm{~mL}$ working volume. The bioreactor was equipped with a thermostat and a gas inlet connected to a helium reservoir and gas outlet. The acclimated DROC and MFP were inoculated with a mixed mesophilic culture that was used as a starter for the anaerobic step. The mixed culture was derived from activated urban sludge. Before starting fermentation the medium was purged for $20 \mathrm{~min}$ with helium to eliminate any trace of dissolved oxygen. The mixture was kept under continuous gentle stirring for $27 \mathrm{~h}$ at $37^{\circ} \mathrm{C}$.

At the end of this time the digested stream was centrifuged at $8,000 \mathrm{rpm}$ for $10 \mathrm{~min}$ to remove solid residues, and sterilized by passing the stream through a polymeric membrane with a pore size of $0.2 \mu \mathrm{m}$. In the case of DROC, the resulting medium was diluted to the desired concentration $(20 \%, 40 \% \mathrm{v} / \mathrm{v})$ with sterile distilled water, derived from membrane process. The $\mathrm{pH}$ was adjusted to 7.2 by adding dropwise a concentrated solution of $\mathrm{NH}_{4} \mathrm{OH}$ under sterile conditions. The diluted medium $(20 \%$ and $40 \% \mathrm{v} / \mathrm{v})$ was used as substrate for the aerobic digestion by $A$. vinelandii UWD that was inoculated at a concentration of $10^{6}$ cells $/ \mathrm{mL}$, after being pre-grown in the same liquid stream. The aerobic digestion was carried out in $2 \mathrm{~L}$ flasks with $500 \mathrm{~mL}$ working volume at $28{ }^{\circ} \mathrm{C}$ under moderate shaking $(150 \mathrm{rpm})$ for desired time (typically 24-150 h) to maximize intracellular PHB accumulation.

Cell vitality was monitored using CFU method. At various interval times $(0,21,46,72 \mathrm{~h})$, an aliquot (100 $\mu \mathrm{L}$ ) of the aerobic fermented medium was removed and distributed onto solid N-free Burk's medium. The plates were incubated at $28{ }^{\circ} \mathrm{C}$ for $72 \mathrm{~h}$. At the end of incubation time the grown colonies has been counted. Growth was also determined photometrically at 600 nm.

The same procedure for the aerobic digestion was used for PHB production in diauxic Burk's medium containing $3 \%(\mathrm{w} / \mathrm{v})$ glucose, $15 \mathrm{mM}$ ammonium acetate and $23 \mathrm{mM} \mathrm{NH} \mathrm{Nl}_{4} \mathrm{Cl}$. The $\mathrm{pH}$ was adjusted to 7.2 with $0.1 \mathrm{~N} \mathrm{NaOH}$. 


\subsection{PHB Extraction}

Intracellular PHB was extracted by using a literature procedure with some minor modifications [24]. At the end of aerobic step, microorganisms were collected by centrifugation at $10,000 \mathrm{rpm}$ for $10 \mathrm{~min}$ at room temperature, and then washed with PBS (phosphate buffered saline), $\mathrm{pH}$ 7.4. The obtained biomass was dried and weighted. The dried biomass was then dissolved in a solution of pure chloroform and sodium hypochlorite 6\% 1:1 v/v (Sigma-Aldrich) using $25 \mu \mathrm{L}$ of solution per mg of pellet; the resulting mixture was kept at $30{ }^{\circ} \mathrm{C}$ overnight under continuous vigorous stirring. Then the solution was then centrifuged at $10,000 \mathrm{rpm}$ for $10 \mathrm{~min}$ at room temperature and the supernatant taken off to isolate the organic fraction, containing PHB. Bio-polymer films were obtained by slow evaporation in air at room temperature in a glass Petri dish. Optionally purified PHB granules were obtained by re-crystallization from chloroform/methanol solution.

\subsection{PHB Characterization}

Nuclear Magnetic Resonance ( ${ }^{1} \mathrm{H}$ NMR) spectra were recorded in deuterated chloroform $\mathrm{CDCl}_{3}$ purchased from Sigma-Aldrich, by using a $600 \mathrm{MHz}$ Bruker Avance II Ultrashield Plus spectrometer. MestReNova software was used to process NMR spectra.

DSC (different scanning calorimetry) was performed on DSC1 Stare System (Mettler Toledo) and was employed to assess the transition temperature $\left(\mathrm{T}_{\mathrm{g}}\right)$ and the melting temperature $\left(\mathrm{T}_{\mathrm{m}}\right)$ of PHB powder. The sample was left in steady condition at $-20^{\circ} \mathrm{C}$, at later stage heated from $-20{ }^{\circ} \mathrm{C}$ to $190{ }^{\circ} \mathrm{C}$ at a constant rate of $10{ }^{\circ} \mathrm{C} / \mathrm{min}$, left at $190{ }^{\circ} \mathrm{C}$ in steady condition and consecutively cooled from $190{ }^{\circ} \mathrm{C}$ to $-20{ }^{\circ} \mathrm{C}$ at a constant rate $50{ }^{\circ} \mathrm{C} / \mathrm{min}$. Finally it was heated from $-20{ }^{\circ} \mathrm{C}$ at $450{ }^{\circ} \mathrm{C}$ at a constant rate of $10{ }^{\circ} \mathrm{C} / \mathrm{min}$. Measurements were carried out under $50 \mathrm{~mL} / \mathrm{min}$ of $\mathrm{N}_{2}$ flow. The PHB powders were weighed (5-15 mg) and introduced into the aluminum pans. Different sample types were run in triplicate.

TGA (Thermogravimetric analysis) of PHB powder was conducted on TGA Stare system (Mettler Toledo) at a heating rate of $10{ }^{\circ} \mathrm{C} / \mathrm{min}$ and over a temperature range of $25{ }^{\circ} \mathrm{C}$ to $1,000{ }^{\circ} \mathrm{C}$ in nitrogen atmosphere $\left(50{ }^{\circ} \mathrm{C} / \mathrm{min}\right.$ ) to evaluate the degradation temperature $\left(\mathrm{T}_{\mathrm{d}}\right)$. The PHB powder was weighed (5-15 mg) introduced into the aluminum pans and was analyzed in triplicate.

\section{Results and Discussion}

To date, a number of barriers to the techno-economic sustainability and the scale-up of PHA industrial production process make difficult the expansion of PHB on the market. One of the reasons is surely the high cost of raw materials that can be overcome by using waste streams as feedstock in fermentation processes, a hot topic studied from different research group over the last decades and currently under investigation [25]. Among all the potential waste streams as raw material for biopolymer production an interesting wastewater stream is OMW. In order to increase the sustainability of wastewater industrial treatment, a crucial point is the valorization of all its valuable chemical components. High added value compounds in OMW are polyphenols, which can be selectively extracted by the membrane-treatment of OMW and subsequent dephenolization of the effluent. This process allows to obtain a polyphenol-rich concentrate composed mainly of decarboxymethylelenolic acid linked to hydroxytyrosol (3,4-DHPEA-EDA), verbascoside, ( $p$-hydroxyphenyl)-ethanol ( $p$-HPEA) e3,4-(dihydroxyphenyl)-ethanol (3,4-DHPEA) and a residual liquid stream that contains other high-carbon load, i.e. DROC.

\subsection{Membrane System, Polyphenol Extraction and DROC Analysis}

The membrane treatments of OMW produced a ROC with final volumes of about $20 \%$ of the original OMW. The membrane treatments also yielded a 
strong reduction of the OMW organic load, with a drawdown of $98 \%$. The initial OMW COD value was $81,568 \mathrm{mg} / \mathrm{L}$; that of the final concentrate from reverse osmosis was $331,360 \mathrm{mg} / \mathrm{L}$. Also the total reducing sugar content increased from $0.74 \%$ in the starting OMW to $8.4 \%$ in the final ROC. As expected and confirmed by DROC analyses, the sugar content remained unvaried after the selective dephenolization treatment on ROC. The total $\mathrm{N}$ amount was almost equal for OMW and ROC, while the MFP showed a decrease that is likely to be due to the retention of micro-sized N-rich compounds in the microfiltration concentrate. The initial slight acidity of OMW was not significantly affected by the membrane treatment as shown by $\mathrm{pH}$ values reported in Table 1 .

The main phenols found in ROC were the same as those in the OMW, but their concentrations, expressed as total phenols, were fourfold higher in the ROC (Table 2). Among these, the most abundant compounds in the ROC were 3,4-DHPEA-EDA, verbascoside and 3,4-DHPEA whereas only small amounts of $p$-HPEA were present in both the ROC and OMW. The slightly higher increase of 3,4-DHPEA concentration in ROC can be ascribed to a minimal hydrolysis of 3,4-DHPEA-EDA, which normally occurs in OMW during storage. The effectiveness of the dephenolizing treatment was demonstrated by the impossibility to detect the major phenolic components, i.e. 3,4-DHPEA-EDA and verbascoside. Only trace of 3,4-DHPEA were detected in the DROC (Table 2). OMW derived polyphenols have a great potential value, as for instance as additives in food, pharmaceutical and cosmetic industry.

\subsection{Microbial PHB Production}

The production of PHAs from waste with a high concentration of $\mathrm{C}$-sources as VFAs (volatile fatty acids) and reducing sugars has been widely investigated and recently reviewed [26]. Due to its characteristic composition, DROC can be an ideal candidate to be used as feedstock in the production of PHAs by fermentation processes.

In order to ascertain whether a membrane treatment prior to microbial production of PHB represents an advantageous approach for increasing the overall PHB yield, the authors used OMW and its derivatives from the membrane treatment as substrate for anaerobic and successive aerobic fermentation, tuning the different parameters of the two phases.

The anaerobic fermentation aimed to the exploitation of the carbohydrates of the waste streams to generate VFAs rich effluents $[27,28]$, which are the most direct substrates for PHAs production. As demonstrated by Cerrone [19], an incubation time of $27 \mathrm{~h}$ is not sufficient to transform all the sugars in VFAs by mesophilic bacteria.

Table 1 Characteristics of OMW and different stream (MFP, ROC) obtained by membrane system.

\begin{tabular}{lllll}
\hline & $\mathrm{COD}\left(\mathrm{mg} / \mathrm{L} \mathrm{O}_{2}\right)$ & Total $\mathrm{N}(\mathrm{g} / \mathrm{L})$ & $\mathrm{pH}$ & Reducing sugars $(\%)$ \\
\hline OMW & 81,568 & 0.93 & 5 & 0.74 \\
MFP & 77,344 & 0.3 & 4.96 & 1.09 \\
ROC & 331,360 & 0.98 & 5.26 & 8.4 \\
\hline
\end{tabular}

Table 2 Phenolic composition (g/L) of OMW, MFP, ROC and DROC.

\begin{tabular}{llllll}
\hline \multicolumn{5}{c}{ Phenolic composition } \\
\hline & $3,4-D H P E A$ & $p$-HPEA & Verbascoside & 3,4-DHPEA-EDA & Total phenolic addition \\
\hline OMW & $0.5 \pm 0.04$ & $0.1 \pm 0.01$ & $1.0 \pm 0.1$ & $3.2 \pm 0.3$ & $4.9 \pm 0.3$ \\
MFP & $0.6 \pm 0.01$ & $0.1 \pm 0.003$ & $1.1 \pm 0.0$ & $3.2 \pm 0.2$ & $5.0 \pm 0.2$ \\
ROC & $3.2 \pm 0.02$ & $0.4 \pm 0.01$ & $4.7 \pm 0.2$ & $14.3 \pm 0,3$ & $22.6 \pm 0.3$ \\
DROC & $0.15 \pm 0.003$ & n.d. & n.d. & n.d. & $0.15 \pm 0.003$ \\
\hline
\end{tabular}


In the aerobic digestion $A$. vinelandii UWD strain was used. Cerrone and colleagues have recently reported the capability of $A$. vinelandii UWD to accumulate higher quantity of PHB than parent Azotobacter strains from OMW. The authors have firstly examined the capability of $A$. vinelandì UWD to grow and accumulate PHB in DROC, MFP and Burk's media.

Attempts to cultivate this bacterial strain in pure DROC resulted in poor or no growth even over a long incubation time. For this reason it was necessary to operate successive dilutions of DROC with ultrapure water recovered from the reverse osmosis step in order to find the optimal conditions for bacterial growth. DROC was diluted to 20 or $40 \% \mathrm{v} / \mathrm{v}$ and used as medium for PHB production. In each case $A$. vinelandii UWD exhibited growth as well as in diauxic Burk's control medium containing glucose and acetate as carbon source.

By using the CFU method, we monitored the cell vitality of $A$. vinelandii UWD in different media at different incubation times $(0,21,46,72 \mathrm{~h})$. Results in Fig. 1 demonstrate growth of $A$. vinelandii UWD in all fermented media. This finding indicates that the bacterium possesses the ability to utilize a variety of carbon sources that may be different from those which are found in molasses cane, beet sugar and corn syrup, the classical substrates for PHB bacterial production [29]. It is also worth of note that in $40 \%$ diluted DROC

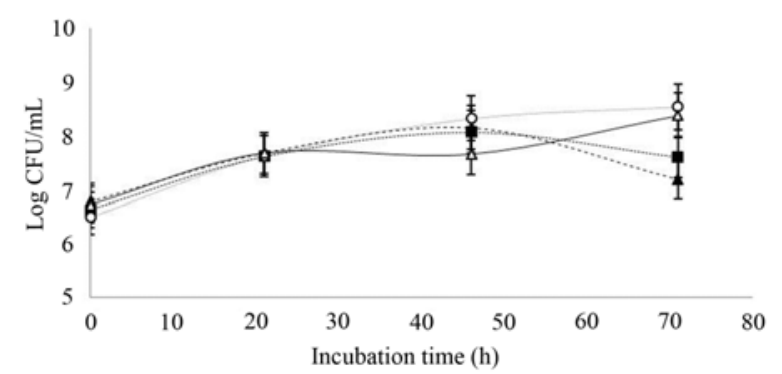

Fig. 1 A. vinelandii UWD growth (expressed as log CFU/ml versus incubation time) in $20 \%$ diluted DROC (- $), 40 \%(\mathrm{O}), 60 \% \operatorname{MFP}\left(\boldsymbol{A}^{-}\right)$and Burk's with $15 \mathrm{mM}$ ammonium acetate, $3 \%$ glucose $(-\square$. it could be observed a logarithmic increase in the number of total aerobic bacteria during $72 \mathrm{~h}$, even higher than in diauxic Burk's medium, indicating that DROC is an optimal substrate for the growth of PHAs-accumulating bacterial strains.

Once the ability of $A$. vinelandii UWD to grow in a variety of media coming from different OMW membrane treatments was demonstrated, the authors processed a complete set of experiments in order to produce and extract PHB. DROC at different concentration $(20 \%$ and $40 \% \mathrm{v} / \mathrm{v})$ and MFP were used as carbon sources for PHB production by aerobic fermentation after an initial anaerobic step, and compared to PHB production from diauxic Burk's medium. The bacterial growth and $\mathrm{PHB}$ production in the aerobic phase was monitored over the time by measuring the optical density (O.D.) at $600 \mathrm{~nm}$ (Fig. 2).

Consistently with previous findings [30-32], A. vinelandii UWD exhibited diauxic growth in the Burk's medium with acetate and glucose. Diauxic growth can be observed when heterotrophic bacteria grow in batch culture on sufficiently high concentrations of more than one carbon and energy source [33]. Analogously to what observed in the Burk's medium with acetate and glucose, in diluted fermented DROC (20\% and $40 \% \mathrm{v} / \mathrm{v})$ the strain showed a similar diauxic behavior during growth,

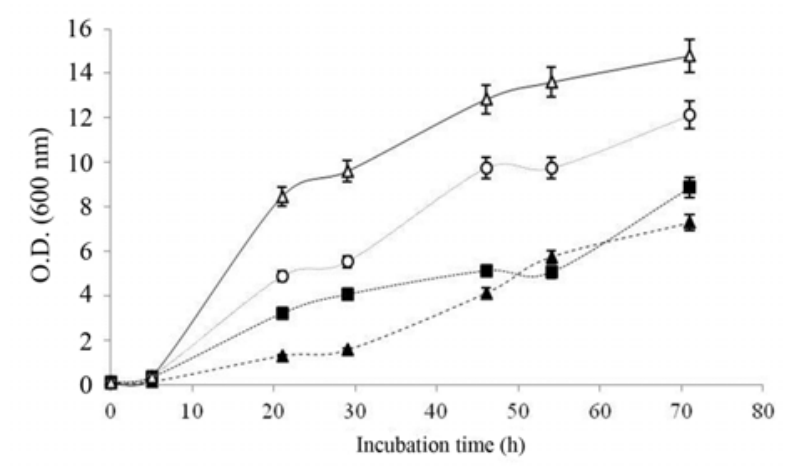

Fig. 2 Growth of $A$. vinelandii UWD (expressed as O.D. versus incubation time) in $20 \%$ diluted DROC (---), $40 \%$ ( $\bigcirc)$, 60\% MFP ( $-\Delta^{-}$) and Burk's with ammonium acetate $15 \mathrm{mM}, 3 \%$ glucose $(\square-$ ). 
indicating that the DROC stream exceeds the minimum concentrations of different carbon sources (VFA and reducing sugars), suitable for bacterial growth and storage of the polymer. In MFP it was more difficult to observe a pronounced diauxic trend, at least from the O.D. measurements, probably due to the inhibitory action of polyphenols on activated sludge during the anaerobic fermentation [34]. It must be highlighted that the O.D. increase is not only related to the growth of bacterial strain during the aerobic fermentation, but also to the accumulation of PHB granules inside the bacterial cells, which causes an increase in light absorbance. This is particularly true at later incubation time points, when PHB storage is more effective $[35,36]$. Thus the differences observed in Fig. 2 are indicative of final yields of PHB production in the different media as confirmed by the data reported below.

It is worth of noting that diluted DROC medium alone is itself a good growth the medium for $A$. vinelandii UWD. As a matter of fact there was no significant difference in PHB yield between diluted DROC medium diluted with Burk's medium salts and water diluted DROC medium. In Figs. 3 and 4 it is possible to compare the growth (CFU/mL) and O.D. increase during $A$. vinelandii UWD aerobic incubation over $72 \mathrm{~h}$ in $20 \%(\mathrm{v} / \mathrm{v})$ diluted DROC in presence or absence of Burk's salts. The curves show that in both cases the bacterium is able to grow and accumulate PHB, as confirmed by the extracted polymer yield, confirming that DROC did not need salt supplement for biopolymer production. Similar results were obtained with 40\% (v/v) diluted DROC (data not shown). The only addition of $\mathrm{NH}_{4} \mathrm{OH}$ solution which has the dual utility of $\mathrm{pH}$ adjustment and nitrogen source suitable for bacterial growth was carried out. Since the DROC diluted media when used as sole feedstock, without the addition of salts, has shown no variation of bacterial growth and yield of the polymer, a reduction of costs with respect to analogous processes which necessitate of initial waste stream composition adjustment can be expected.

Fig. 5 reports the trend of the polymer productivity (i.e. the amount of polymer per unit volume of medium per unit time) after $72 \mathrm{~h}$ of growth in different

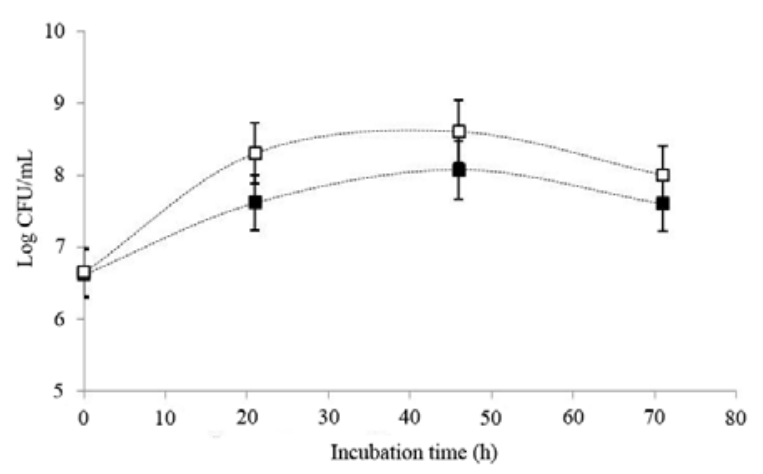

Fig. 3 A. vinelandii UWD growth (expressed as log CFU/ml versus incubation time) in $20 \%$ diluted DROC with Burk's salts $\left({ }^{-\cdot-\cdot--)}\right.$ ) or with sterile distilled water ( - - - - )

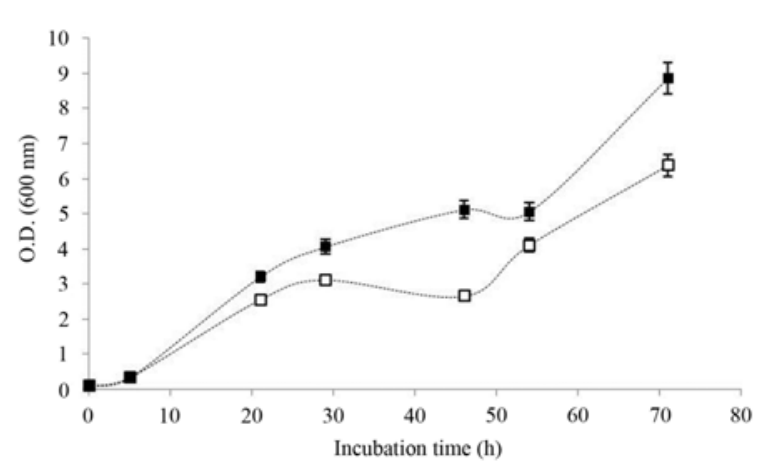

Fig. 4 A. vinelandii UWD growth (expressed as O.D. versus incubation time) in diluted $20 \%$ DROC with Burk's

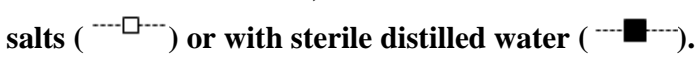

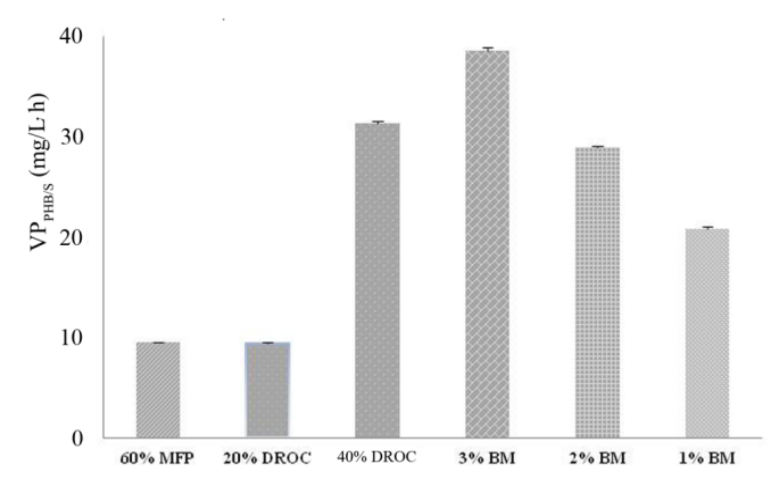

Fig. $5 \mathrm{VP}_{\mathrm{PHB} / \mathrm{S}}($ expressed in $\mathrm{mg} / \mathrm{L} \cdot \mathrm{h}$ ) in $20 \%, 40 \%$ diluted DROC, $60 \%$ diluted MFP, Burk's salts medium (BM) with ammonium acetate $(15 \mathrm{mM})$ and different concentrations of glucose $(1 \%, 2 \%, 3 \%)$. 
media. In these experiments, the Burk's medium was supplemented with different glucose concentrations ranging from $1 \%$ to $3 \%(\mathrm{w} / \mathrm{v})$ keeping constant the ammonium acetate concentration $(15 \mathrm{mM})$. Glucose levels above $3 \%(\mathrm{w} / \mathrm{v})$ were not tested because they could be inhibitory to bacterial growth [31,37].

The results with different DROC concentration $(20 \%, 40 \% \mathrm{v} / \mathrm{v})$ highlighted variable polymer volumetric productivity ( $\mathrm{VP}_{\mathrm{PHB} / \mathrm{S}}$ ) equal to 31.4 $\mathrm{mg} / \mathrm{L} \cdot \mathrm{h}$ of PHB. Lower yield was obtained with $20 \%$ $(\mathrm{v} / \mathrm{v})$ diluted DROC possibly because dilution of carbon sources (VFAs and reducing sugars) was too high to obtain an optimal PHB accumulation. The $\mathrm{VP}_{\mathrm{PHB} / \mathrm{S}}$ of $20 \%$ diluted DROC was comparable to the result obtained with $60 \%$ diluted MFP because both streams had a low concentration of carbon sources.

The advantage of using an integrate system with an initial membrane filtration unit must be evaluated taking into account the characteristics of efficiency of PHB production, working volumes of the fermenter employed, and cost of added components in the culture medium. The uselessness of the addition of salts to DROC has been highlighted above. Moreover the DROC seems to match the requirements of maximizing PHB yields and reducing working volumes if the production process performances are referred to the starting OMW, thus making it a cheaper stream suitable for industrial use. As a matter of fact, as shown in Fig. 5, the efficiency of $\mathrm{VP}_{\mathrm{PHB} / \mathrm{s}}$ is comparable when the same volume of $20 \%$ diluted (v/v) DROC and $60 \%$ diluted (v/v) MFP is used as initial feedstock, but a lower working volume is used for $20 \%$ diluted DROC as reported in Table 3. Moreover, if the authors compare the results obtained with $60 \%$ diluted MFP, that can represent the state of the art reference, with the ones obtained with $40 \%$ diluted ( $/ \mathrm{v})$ DROC an even greater advantage of using the integrated system of membrane filtration + fermentation is evident. In fact, not only it is observed an increase of about 9 times in $\mathrm{VP}_{\mathrm{PHB} / \mathrm{S}}$ for $40 \%$ diluted DROC compared with MFP (Fig. 5), but also a much lower (about 37\%) working volume as summarized in Table 3. This results in an overall increase of PHB productivity of our integrated process from the starting OMW ( $\mathrm{VP}_{\mathrm{PHB} / \mathrm{OMW}}$ ) as shown by the data reported in the Table 3 for $40 \%$ diluted $(\mathrm{v} / \mathrm{v})$ DROC, with a PHB production of $19.6 \mathrm{mg} / \mathrm{L} \cdot \mathrm{h}$.

In other words, considering the MFP results as the benchmark of the state-of-the art process for PHB production, the use of an initial membrane filtration system allows the reduction of fermentation working volumes and at the same time an increase of PHB yields, thus making the integrated system advantageous for the biopolymer production on an industrial scale.

\subsection{PHB Characterization}

The composition of polyester, produced from different streams, was determined by integration of the proton resonances of 3-hydroxybutirate unit in the $\mathrm{H}^{1}$ NMR spectra. The comparison of NMR analysis on pure commercial PHB (Sigma-Aldrich) and PHB extracted and purified from $20 \%$ and $40 \%(\mathrm{v} / \mathrm{v})$ diluted DROC and MFP confirmed the nature of the extracted biopolymer. The characteristic PHB peaks at $\delta=5.2,2.5$ and 1.2 corresponding to $\mathrm{a}-\mathrm{CH}$ doublet,- $\mathrm{CH}_{2}$ multiplet and $\mathrm{CH}_{3}$ doublet respectively were observed [38]. No additional detectable peaks attributable to organic impurities were recorded, thus confirming the effectiveness of the extraction and purification protocols used.

The thermogravimetric analysis were carried out to prove the thermal polymeric characteristics, including glass transition temperature $\left(\mathrm{T}_{\mathrm{g}}\right)$, melting temperature $\left(T_{f}\right)$ and degradation temperature $\left(T_{d}\right)$ of commercial PHB and PHB produced from streams.

Table 3 Working volume of fermenter and performance of PHB on OMW.

\begin{tabular}{lll}
\hline Stream & $\begin{array}{l}\text { Working volume } \\
(\mathrm{L})\end{array}$ & $\begin{array}{l}\mathrm{VP}_{\text {PHB/OMW }} \\
(\mathrm{mg} / \mathrm{L} \cdot \mathrm{h})\end{array}$ \\
\hline $20 \%$ diluted DROC & 1.25 & 11.75 \\
$40 \%$ diluted DROC & 0.62 & 19.62 \\
MFP & 1.66 & 15.77 \\
\hline
\end{tabular}


The data showed that degradation of commercial PHB occurs at slightly lower temperature with respect to PHBs extracted and purified from $20 \%$ and $40 \%$ (v/v) diluted DROC and MFP, indicating that the PHB produced from DROC and MFP streams presents a better thermal stability. On the other hand no significant differences on $\mathrm{T}_{\mathrm{g}}$ and $\mathrm{T}_{\mathrm{m}}$ between commercial PHB and PHB produced and extracted from DROC and MFP streams were observed by DSC analyses (data not shown).

It is worth noting that the PHB produced and extracted from A. vinelandii UWD grown up in different streams as $20 \%$ and $40 \%(\mathrm{v} / \mathrm{v})$ diluted DROC and MFP, exhibited the same values for $T_{g}, T_{m}$ and $T_{d}$.

The majority of PHA production processes from OMW affords a final variable product as mixed bacterial cultures are used, which produces and accumulates different polyhydroxyalkanoate co-polymer, usually PHB-HV (poly-3-hydroxybutyrate-co-hydroxyvalerate) with a highly variable hydroxyvalerate content ranging from 6 to $14 \%$ [16]. On the contrary the authors opted for the use a pure culture of $A$. vinelandii UWD [39] in the aerobic phase, useful to produce a polymer that is not subject to structural variability, i.e. poly-3-hydroxybutyrate homopolymer. As mentioned above the PHB polymer produced by A. vinelandii UWD shows thermal properties independent on the dilution of the DROC with ultrapure water, while the fine control of the waste feedstock composition in mixed culture PHA-production approach is crucial for the final chemical nature of the product and consequently for its thermal behavior. The only constrain of the process is obviously represented by the range of DROC dilution necessary to ensure the bacteria growth and accumulation. As a matter of fact DROC concentration higher than $50 \%$ seems to have an inhibitory action on the production of polymer as confirmed by failure of attempting PHB production in $60 \%, 80 \%$ and $100 \%(\mathrm{v} / \mathrm{v})$ diluted DROC. The membrane filtration system thus offer a better control on the natural variability of the initial waste feedstock (OMW) combined with the major versatility of a pure-culture bacterial aerobic fermentation for a more standardized PHB product.

Moreover the use of diluted DROC as feedstock instead of diluted MFP, which represent the state of the art benchmark, presents some additional advantages in terms of final product properties. Although A. vinelandii UWD strain always accumulates in diauxic conditions a polymer with the same thermal profile, the thermal behavior of the PHB extracted from different streams presented some differences if the samples were subjected to an annealing at $190{ }^{\circ} \mathrm{C}$ for $5 \mathrm{~min}$. This treatment leads to the appearance of a peak at about $162{ }^{\circ} \mathrm{C}$ next to the original $\mathrm{T}_{\mathrm{m}}$ peak in the DSC curves, that can be associated to the melting of lower molecular weight PHB derived from a random scission of the starting long chain polymer, caused by the annealing at $\mathrm{T}>\mathrm{T}_{\mathrm{m}}$ [40]. This behavior was more evident for all the samples produced from the MFP stream, indicating the PHB produced from the latter is more susceptible to chain fragmentation upon treatments at temperatures close to the $T_{m}$ than the PHB produced from DROC streams. As a matter of fact the peak recorded at $162{ }^{\circ} \mathrm{C}$ in the DSC thermogram after annealing the sample at $190{ }^{\circ} \mathrm{C}$ is higher in the case of PHB produced from MFP than the one produced from $40 \%$ DROC (Fig. 6). This

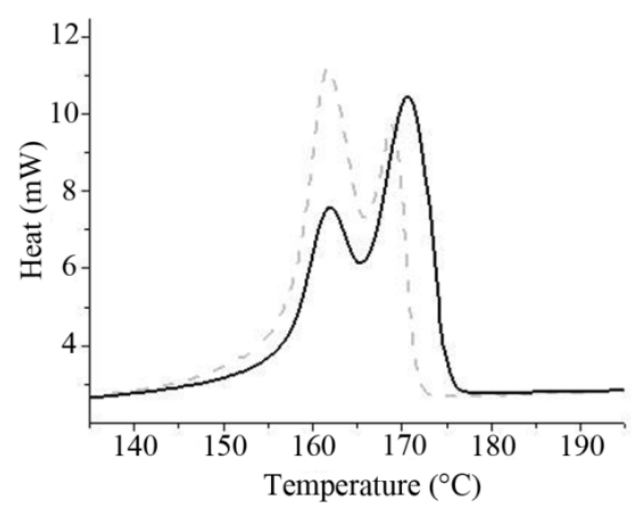

Fig. 6 Details of DSC curves of PHB obtained from $40 \%$ DROC (straight line) and from 60\% MFP (dashed line) for the melting temperature recorded after annealing the sample at $190{ }^{\circ} \mathrm{C}$ for $5 \mathrm{~min}$. 
feature suggests that the latter biopolymer is more suitable for the conventional high-temperature (extrusion, molding etc.) plastic manufacturing technologies.

\section{Conclusions}

The integration of membrane filtration techniques in addition to bacterial PHB production from OMW allows a higher yield of the bio-polymer (two fold in the best case) than the one obtained with simple OMW fermentation. The resulting integrated process presents thus three main advantages: the simultaneous recovery of added value polyphenols from OMW, the increase of PHB productivity, and the reduction of fermentation working volumes. The low energy consumptions and the high costs currently required for OMW disposal and make the process here reported truly cost-effective, as confirmed by forthcoming reports on LCA and specific economic aspects. This approach is thus suitable for a large scale PHB production from wastewater.

\section{References}

[1] Koller, M., Atlic, A., Dios, M., Reiterer, A., and Braunegg, G. 2010. "Microbial PHA Production from Waste Raw Materials." In Plastic from Bacteria: Natural functions and Applications, edited by Chen, G. Q. Berlin Heidelberg: Springer-Verlag.

[2] Chanprateep, S. 2010. "Current Trends in Biodegradable Polyhydroxyalkanoates." Journal of Bioscience and Bioengineering 110: 621-32.

[3] Du, C., Sabirova, J., Soetaert, W., and Lin, S. K. C. 2012. "Polyhydroxyalkanoates Production from Low-Cost Sustainable Raw Materials." Current Chemical Biology 6: 14-25.

[4] Suwannasing, W., Moonamart, S., and Kaewkannetra, P. 2011. "Yields of Polyhydroxyalkanoates (PHAs) during Batch Fermentation of Sugar Cane Juice by Alcaligenes latus and Alcaligeneseutrophus." Journal of Life Sciences 5: 960-6.

[5] Chua, A. S. M., Takabatake, H., Satoh, H., and Mino, T. 2003. "Production of Polyhydroxyalkanoates (PHA) by Activated Sludge Treating Municipal Wastewater: Effect of $\mathrm{pH}$, Sludge Retention Time (SRT), and Acetate Concentration in Influent." Water Research 37: 3602-11.

[6] Dionisi, D., Carucci, G., Papini, M. P., Riccardi, C.,
Majone, M., and Carrasco, F. 2005. "Olive Oil Mill Effluents as a Feedstock for Production of Biodegradable Polymers." Water Research 39: 2076-84.

[7] Servili, M., Esposto, S., Veneziani, G., Urbani, S., Taticchi, A., Di Maio, I., Selvaggini, R., Sordini, B., and Montedoro, G. 2011. "Improvement of Bioactive Phenol Content in Virgin Olive Oil with an Olive-Vegetation Water Concentrate Produced by Membrane Treatment." Food Chemistry 124: 1308-15.

[8] Niaounakis, M., and Halvadakis, C. P. 2004. Olive-Mill Waste Management: Literature Review and Patent Survey. Atene: Typothito publications.

[9] Morillo, J. A., Aguilera, M., AntizarLadislao, B., Fuentes, S., Ramos-Cormenzana, A., Russell, N. J., and Monteoliva-Sanchez, M. 2008. "Molecular Microbial and Chemical Investigation of the Bioremediation of Two-Phase Olive Mill Waste Using Laboratory-Scale Bioreactors." Applied Microbiology and Biotechnology 79: 309-17.

[10] Ntougias, S., Bourtzis, K., and Tsiamis, G. 2013. "The Microbiology of Olive Mill Wastes." BioMed Research International 2013: 1-16.

[11] Karpouzas, D. G., Ntougias, S., Iskidou, E., Rousidou, C., and Papadopoulou, K. K. 2010. "Olive Mill Wastewater Affects the Structure of Soil Bacterial Communities." Applied Soil Ecology 45: 101-1.

[12] Carraro, L., Fasolato, L., Montemurro, G., Martino, M. E., Balzan, S., Servili, M., Novelli, E., and Cardazzo, B. 2014. "Polyphenols from Olive Mill Waste Affect Biofilm Formation and Motility in Escherichia coli K-12." Microbial Biotechnology 7: 265-75.

[13] Garcia-Castello, E., Cassano, A., Criscuoli, A., Conidi, C., and Drioli, E. 2010. "Recovery and Concentration of Polyphenols from Olive Mill Wastewaters by Integrated Membrane System." Water Research 44: 3883-92.

[14] Mudimu, O. A., Peters, M., Brauner, F., and Braun, G. 2012. "Overview of Membrane Processes for the Recovery of Polyphenols from Olive Mill Wastewater." American Journal of Environmental Sciences 8: 195-201.

[15] Borja, R., Rincón, B., and Raposo, F. 2006. “Anaerobic Biodegradation of Two-Phase Olive Mill Solid Wastes and Liquid Effluents: Kinetic Studies and Process Performance." Journal of Chemical Technology and Biotechnology 81: 1450-62.

[16] Campanari, S., e Silva, F. A., Bertin, L., Villano, M., and Majone, M. 2014. "Effect of the Organic Loading Rate on the Production of Polyhydroxyalkanoate in a Multi-stage Process Aimed at the Valorization of Olive Oil Waste Water." International Journal of Biological Macromolecules 71: 34-41.

[17] Ntaikou, I., Valencia Pedoni, C., Kouurmentza, C., Ilieva, 
V. I., Morelli, A., Chiellini, E., and Lyberatos, G. 2014. "Microbial Bio-based Plastics from Olive-Mill Wastewater: Generation and Properties of Polyhydroxyalkanoates from Mixed Cultures in a Two-Stage Pilot Scale System.” Journal of Biotechnology 188: 138-47.

[18] Beccari, M., Bertin, L., Dionisi, D., Fava, F., Lampis, S., Majone, M., Valentino, F., Vallini, G., and Villano, M. 2009. "Exploiting Olive Oil Mill Effluent as a Renewable Resource for Production of Biodegradable Polymers Through a Combined Anaerobic-aerobic Process." Journal of Chemical Technology and Biotechnology 84: 901-8.

[19] Cerrone, F., Sánchez-PeinadoMde, M., Juárez-Jimenez, B., González-López, J., and Pozo, C. 2010. "Biological Treatment of Two-phase Olive Mill Wastewater (TPOMW, alpeourujo): Polyhydroxyalkanoates (PHAs) Production by Azotobacter Strains." Journal of Microbiology and Biotechnogy 20: 594-601.

[20] Pozo, C., Martínez-Toled, M. V., Rodelas, B., González-López, J. 2002. "Effects of Culture Conditions on the Production of Polydroxyalkanoates by Azotobacterchroococcum $\mathrm{H} 23$ in Media Containing a High Concentration of Alpechín (Wastewater from Olive Oil Mills) as Primary Carbon Source." Journal of Biotechnology 97: 125-31.

[21] Eaton, A. D., Clesceri, L. S., Rice, E. W., Greenberg, A. E., and Franson, M. A. H. 2005. Standard Methods for the Examination of Water and Wastewater. Washington, DC: Centennial.

[22] Nielsen, S. S. 2010. Food Analysis Laboratory Manual. New York: Springer.

[23] Servili, M., Selvaggini, R., Taticchi, A., and Montedoro, G. F. 2001. "Headspace Composition of Virgin Olive Oil Evaluated by Solid Phase Microextraction: Relationship with the Oil Sensory Characteristics." In Food Flavorus and Chemistry: Advances of the New Millennium, edited by Spanier, A. M., Shahidi, F., Parliament, T. H., Mussinan, C., Ho, C. T., and Tratratas Contis, E. United Kindom: The Royal society of Chemistry Publisher.

[24] Singh, P., and Parmar, N. 2011. "Isolation and Characterization of Two Novel Polyhydroxybutyrate (PHB)-Producing Bacteria." African Journal of Biotechnology 10: 4907-19.

[25] Heimersson, S., Morgan-Sagatsume, F., Peters, G. M., Werker, A., and Svantrom, M. 2014. "Methodological Issues in Life Cycle Assessment of Mixed-Culture Polydroxyalkanoate Production Utilizing Waste as Feedstock." New Biotechnology 31: 383-93.

[26] Saharan, B. S., Grewal, A., and Kumar, P. 2014. "Biotechnological Production of Polyhydroxyalkanoates:
A Review on Trends and Latest Developments." Chinese Journal of Biology 2014: 1-18.

[27] Frandinho, J. C., Oehmen, A., and Reis, M. A. M. 2014. "Photosynthetic Mixed Culture Polyhydroxyalkanoate (PHA) Production from Individual and Mixed Volatile Fatty Acids (VFAs): Substrate Preferences and Co-substrate Uptake." Journal of Biotechnology 185: 19-27.

[28] Lee, W. S., Chua, A. S. M., Yeoh, H. K., and Ngoh, G. C. 2014. "A Review of the Production and Applications of Waste-Derived Volatile Fatty Acids." Chemical Engineering Journal 235: 83-99.

[29] Tan, G. Y. A., Chen, C. L., Li, L., Ge, L., Wang, L., Razaad, I. M. N., Li, Y., Zhao, L., Mo, Y., and Wang, J. Y. 2014. "Start a Research on Biopolymer Polyhydroxyalkanoate (PHA): A Review." Polymers 6: 706-54.

[30] Page, W. J., and Knosp, O. 1989. "Hyperproduction of Poly- $\beta$-hydroxybutyrate during Exponential Growth of Azotobactervinelandii UWD.” Applied Environmental Microbiology 55: 1334-9.

[31] Tauchert, K., Jana, A., and Oelze, J. 1990. "Control of Diauxic Growth of Azotobactervinelandii on Acetate and Glucose." Journal of Bacteriology 172: 6447-51.

[32] Chen, G. C., and Page, W. J. 1997. "Production of Poly- $\beta$-hydroxybutyrate by Azotobactervinelandii in Two-Stage Fermentation Process." Biotechnology Technique 11: 347-50.

[33] Harder, W., and Dijkhuizen, L. 1982. "Strategies of Mixed Substrate Utilization in Microorganisms." Philosophical Transactions of the Royal Society of London. Series B, Biological Sciences 297 (6): 459-80.

[34] Bubonja-Sonje, M., Giacometti, J., and Abram, M. 2011. "Antioxidant and Antilisterial Activity of Olive Oil, Cocoa and Rosemary Extract Polyphenols." Food Chemistry 127: 1821-7.

[35] Page, W. J., Manchak, J., and Rudy, B. 1992. "Formation of Poly-(hydroxybutyrate-co-hydroxyvalerate) by Azotobactervinelandii UWD." Applied Environmental Microbiology 58: 2866-73.

[36] Tian, J., Aimin, H., Lawrence, A. G., Liu, P., Watson, N., Sinskey, A. J., and Stubbe, J. 2005. "Analysis of Transient Polyhydroxybutyrate Production in Wautersiaeutropha H16 by Quantitative Western Analysis and Transmission Electron Microscopy." Journal of Bacteriology 187: 3825-32.

[37] George, S. E., Costenbader, C. J., and Melton, T. 1985. "Diauxic Growth in Azotobactervinelandii." Journal of Bacteriology 164: 866-71.

[38] Bluhm, T. L., Hamer, G. K., Marchessault, R. H., Fyfe, C. A., and Veregin, R. P. 1986. "Isodimorphism in Bacterial 


\section{Poly-3-hydroxybutyrate Production}

Poly ( $\beta$-hydroxybutyrate-co- $\beta$-hydroxyvalarate)." Macromolecules 19: 2871-6.

[39] Díaz-Barrera, A., and Soto, E. 2010. "Biotechnological Uses of Azotobactervinelandii: Current State, Limits and Prospects.” African Journal of Biotechnology 9: 5240-50.
[40] Janigova, I., Lacik, I., and Chodak, I. 2002. "Thermal Degradation of Plasticized Poly-(3-hydroxybutyrate) Investigated by DSC." Polymer Degradation and Stability 77: 35-41. 